\title{
Fetal Movements
}

\section{Kazuo Maeda*}

Department of Obstetrics and Gynecolgoy (Honorary Professor), Tottori University Medical School, Yonago, Japan

Fetal movements had been detected by the maternal perception of "kicking" of the fetus, which was the sign of fetal well-being, i.e. Mothers worried about the reduction of fetal kicking, The kicking count in a definite time was a fetal monitoring. However, since the mother percepted fetal movement when it was conducted to the surface of maternal abdomen, the number of maternal perception was only $28 \%$ of the recorded fetal movements by using direct detection of fetal movements with actocardiogram [1]. In addition there may be a false positive kicking when the fetus repeats the hiccupping movements and the mother percepts them. Manning's biophysical profile score (BPPS) [2] and Nijhuis's fetal behavior [3] utilized real-time ultrasonic B-mode, where the image was displayed on the monitor screen, and the observer decided it was fetal movement, and the event was recorded on he on the chart by observer's hand. It is better than maternal perception, but did not suitable to prolonged study on the alternative appearance of fetal active and resting states. Although the BPP report said that the loss of FHR acceleration in non-reactive FHR pattern is abnormal, the acceleration is lost in the resting fetal state, and the state is unable to be differentiated from non-reactive FHR pattern in simple record of FHR in cardotocogram (CTG), i.e. both resting fetal state and nonreactive pattern are the state of no acceleration, while the non-reactive pattern is associated with active fetal movements (bursts), while there is no fetal movement burst in resting fetal state of normal fetus (Figure 1). Both states must be differentiated, because a true non-reactive FHR is followed by severe fetal asphyxia some days later [4]. Although the physiologic sinusoidal FHR should be differentiated from the very ominous true sinusoidal FHR pattern, simple FHR trace was unable to differentiate physiologic sinusoidal of normal outcome from the ominous true sinusoidal FHR, while physiological sinusoidal FHR is accompanied by periodic repeating fetal movements, which synchronize the physiological sinusoidal heart rate [5], i.e. the physiologic sinusoidal heart rate is provoked by the sinusoidal fetal movements. 2) True sinusoidal FHR did not accompany fetal movements. From these facts, simultaneous recording of fetal movement signal and FHR was clinically requested.

Maeda et al. [6] produced a mechanical fetal actograph recorded at maternal abdomen, while its sensitivity was low, and only fetal movements reached to abdominal surface was recorded, therefore

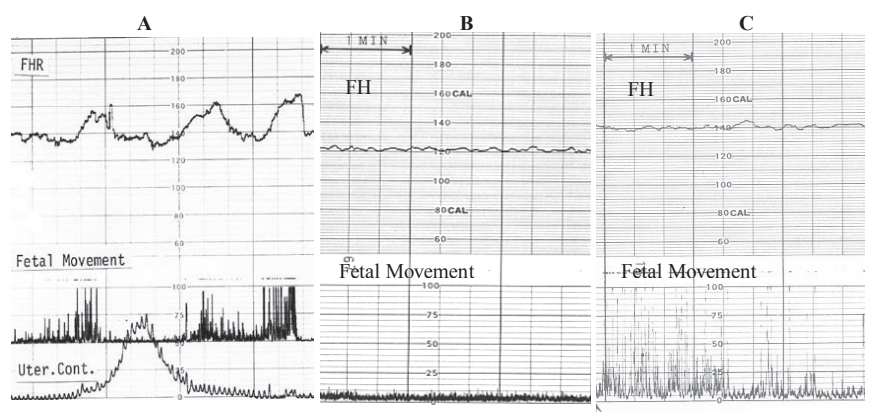

Figure 1: Actocardiograms. A: Active fetal state. Multiple FHR accelerations provoked by fetal movement bursts.B: Resting fetal state. No FHR acceleration and no fetal movement burst, preserving baseline variability. C: Non-reactive FHR pattern. No FHR acceleration against active fetal movement bursts. It was followed by severe fetal asphyxia some days later. it was discarded. In order to directly record fetal movement, the ultrasonic Doppler fetal movement signal was investigated, i.e. a TOITU ultrasonic Doppler FHR monitor was modified by the author to detect fetal movement Doppler signal using the same ultrasonic probe. The Doppler signal of fetal heart beats (higher than $100 \mathrm{~Hz}$ ) was separated from movement signal $(20-50 \mathrm{~Hz}$, when the ultrasound was $2 \mathrm{MHz}$ ) by a band-pass filter, then heart signal was introduced in autocorrelation FHR meter, and movement signal was changed to spikes, and both FHR and movement signals were simultaneously traced on a CTG chart [7]. Active and resting fetal states appeared alternatively, the hyperactive fetal state was characterized by long fetal movements, the resting fetal state was separated from non-reactive FHR pattern, physiologic sinusoidal FHR pattern was diagnosed by periodic and continuous fetal respiratory movements, fetal hiccupping movements, and other fetal chest movements conducted from fetal suction movements and extremity movements were recorded. All fetal movements were recorded by the ultrasonic Doppler actocardiogram in the reports of researchers, except for fetal face expression, which was reported to indicate the risk of antenatal fetus using Kurjak's KANET index of the fetus with $4 \mathrm{D}$ ultrasound [8].

Triangular FHR acceleration was provoked by fetal movement burst in the mid-brain of fetal brain. Long term FHR variability developed by the stimulation of minor fetal movement also in the mid-brain, which was preserved in the non-reactive FHR and lost in the severe fetal brain damage similarly to the loss of variability in anencephalic fetus, i.e. the loss of FHR variability is the sign of fetal brain damage, which may be followed by cerebral palsy (CP), thus it is beneficial to perform cesarean section before the loss of baseline variability further to reduce CP [9]. The acceleration duration ratio to the duration of fetal movement burst

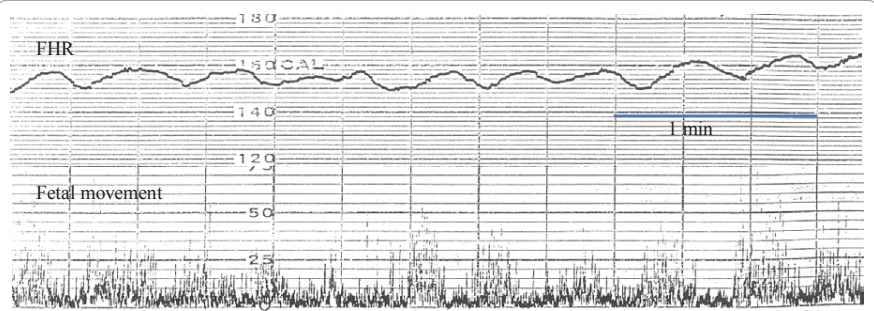

Figure 2: Physiologic benign sinusoidal FHR patter, diagnosed by simultaneously recorded periodic fetal movements, which would provoke moderate FHR variability.

*Corresponding author: Kazuo Maeda, Department of Obstetrics and Gynecology (Emeritus), Tottori University Medical School, Yonago, Japan, 3-125, Nadamachi, Yonago, Tottoriken, 6830835, Japan, Tel: 81-859-22-6856; Fax: 81-859-22-6856; E-mail:maeda@mocha.ocn.ne.jp

Received October 22, 2013; Accepted October 23, 2013; Published October 28 2013

Citation: Maeda K (2014) Fetal Movements. J Health Med Informat 5: e115. doi:10.4172/2157-7420.1000e115

Copyright: ( 2014 Maeda K. This is an open-access article distributed under the terms of the Creative Commons Attribution License, which permits unrestricted use, distribution, and reproduction in any medium, provided the original author and source are credited. 
(A/B ratio) closely correlated to the severity of fetal central nervous system and common fetal disorders indicating the damages of short and long-term outcome, where decreasing A/B ratio showed proportional worsening of fetal outcome [10]. These diagnostic techniques had been unable by the simple FHR analysis.

Minor fetal movement signals recorded on the fetal movement trace of ACG was suppressed after the appearance of large transient fetal bradycardia, indicating the paralysis of fetal motion center, and the minor movement disappeared in the severe fetal asphyxia accompanied by the loss of variability immediately prior to fetal demise. It may also accompany the loss of FHR acceleration, where the loss of fetal movement is an ominous sign, and therefore, continuous disappearance of fetal movement will be diagnosed as severe fetal depression in nonreassuring fetal status developed before fetal demise.

\section{References}

1. Ohta M (1985) Evaluation of fetal movements with ultrasonic Doppler fetal actograph. Nihon SankaFujinkaGakkaiZasshi 37: 73-82.

2. Manning FA, Morrison I, Lange IR, Harman CR, Chamberlain PF (1987) Fetal biophysical profile scoring: selective use of nonstress test. Am J Obstet Gynecol 156: 709-712.
3. Nijhuis JG (1993) Fetal behavioral states. In FA Chervnak, GC Isaacson, S Campbell eds. Ultrasound in Obstetrics and Gynecology, Vol 1, Boston, Little \& Brown, 447-445

4. Teshima N (1993) Non-reactive pattern diagnosed by ultrasonic Doppler feta actocardiogram and outcome of the fetus with non-reactive pattern. Nihon Sanka Fujinka Gakkai Zasshi 45: 423-430.

5. Ito T, Maeda K, Takahashi H, Nagata N, Nakajima K, et al. (1994) Differentiation between physiologic and pathologic sinusoidal FHR pattern by fetal actocardiogram. J Perinat Med 22: 39-43.

6. Maeda K, Kimura S, Nakano H (1969) Pathophysiology of fetus. Fukuoka Printing, Fukuoka.

7. Maeda K (1984) Studies on new ultrasonic Doppler fetal actograph and continuous recording of fetal movement. Acta Obstet Gynecol Jpn 86: 280-288.

8. Talic A, Kurjak A, Stanojevic M (2012) The assessment of fetal brain function in fetuses with ventriculomegaly.: the role of KANET test. J Matern Fetal Neonatal Med 25: 1267-1272.

9. Maeda K (2012) Actocardiographic analysis of fetal hypoxia detected by the bradycardia, loss of fetal heart rate acceleration and long term variability. $J$ Health Medical Informatics.

10. Maeda K, Iwabe T, Yoshida S, Ito T, Minagawa Y, et al. (2009) Detaildmultigrade evaluation of fetal disorders with the quantified actocardiogram. J Perinat Med 37: 392-396. 